\title{
Understanding the centrality deficit: Insight from foreign language learners
}

\author{
Amanda C. Miller • Janice M. Keenan
}

Published online: 23 December 2010

(C) Psychonomic Society, Inc. 2010

\begin{abstract}
This study replicated and extended a phenomenon in the text memory literature referred to as the centrality deficit Miller \& Keenan (Annals of Dyslexia 59:99-113, 2009). It examined how reading in a foreign language (L2) affects one's text representation and ability to recall the most important information. Readers recalled a greater proportion of central than of peripheral ideas, regardless of whether reading in their native language (L1) or a foreign language (L2). Nonetheless, the greatest deficit in participants' L2 recalls, as compared with L1 recalls, was on the central, rather than the peripheral, information. This centrality deficit appears to stem from resources being diverted from comprehension when readers have to devote more cognitive resources to lower level processes (e.g., L2 word identification and syntactic processing), because the deficit was most evident among readers who had lower L2 proficiency. Prior knowledge (PK) of the passage topic helped compensate for the centrality deficit. Readers with less L2 proficiency who did not have PK of the topic displayed a centrality deficit, relative to their $\mathrm{L} 1$ recall, but this deficit dissipated when they did possess PK.
\end{abstract}

Keywords Reading comprehension - Memory for text . Foreign language processing $\cdot$ Prior knowledge

It is a well-known finding in the text memory literature that the more central an idea is to the gist of a text, the more likely it is to be recalled (Britton, Meyer, Hodge, \& Glynn, 1980; Brown \& Smiley, 1978; Cirilo \& Foss, 1980; Curran

A. C. Miller $(\bowtie) \cdot J$. M. Keenan

Department of Psychology, University of Denver,

2155 S. Race St.,

Denver, CO 80208, USA

e-mail: miller23@gmail.com et al. 1996; Keenan \& Brown, 1984; Kintsch \& Keenan, 1973; Kintsch, Kozminsky, Streby, McKoon, \& Keenan, 1975; Kintsch \& van Dijk, 1978; Meyer, 1974; Miller \& Keenan, 2009). Retellings of text show a well-established effect of centrality such that recall monotonically increases as the level of centrality of the idea increases-what has been referred to as the centrality effect.

As we read, we form connections among the semantically related text ideas and, thereby, develop a connected representation of the text. Ideas having many connections emerge from the text representation as being central, while those with fewer connections emerge as peripheral. The centrality effect in text recall is thought to occur because the reader is able to best recall the ideas with the greatest number of connections, which are the central ideas; the peripheral information, with fewer connections, is less likely to be recalled. Miller and Keenan (2009) illustrated that comparing the centrality effects of two groups of readers can be very informative. They found that both good and poor readers showed the familiar centrality effect: Recall increased as the centrality of the information increased. Most interestingly, although good readers recalled more central and peripheral information than did poor readers, the difference between the groups was actually larger for the central information than for the peripheral information (see the top panel of Fig. 1, which shows a significant interaction between reading ability and centrality). Miller and Keenan referred to this greater deficit for central information as the centrality deficit.

The centrality deficit is a counterintuitive finding: The ideas best recalled by poor readers (i.e., central information) are also the source of their greatest deficit, as compared with good readers. It seems more intuitive either that those who struggle to decode words would show a deficit, relative to good readers, across the board (for both central and peripheral ideas) or that they would get the main ideas 


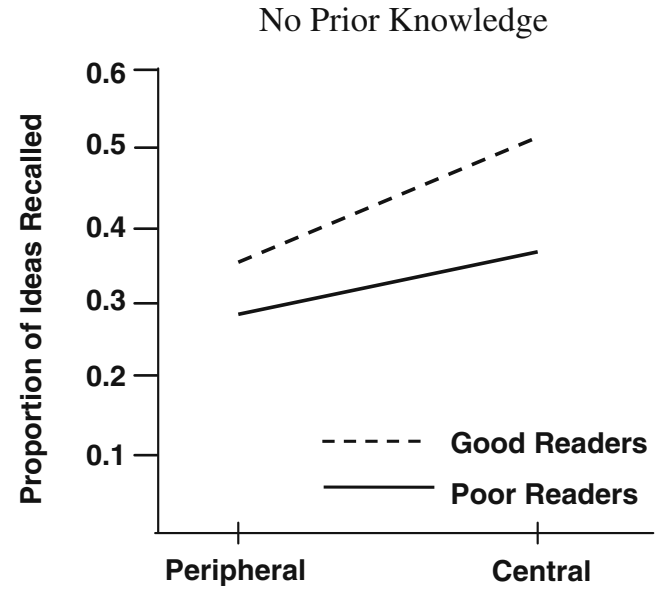

High Prior Knowledge

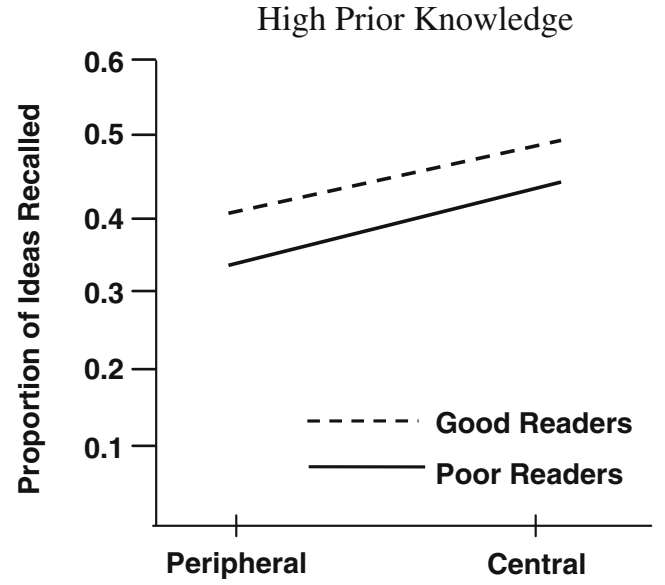

Fig. 1 Proportions of central and peripheral ideas recalled by children who were good readers versus poor readers, with and without prior knowledge of the passage topic. Means are adjusted for IQ and listening comprehension (adapted from Miller \& Keenan, 2009)

and show a deficit only in recalling the less critical information. The centrality deficit can be explained, however, by realizing that it is an indication of a failure to build a coherent representation of the text. Because poor readers must devote a greater proportion of their cognitive resources to lower level processes such as word identification, they consequently have fewer resources remaining for forming connections among the text's ideas that allow centrality to emerge to the same degree that it does for good readers. Central ideas emerge as central because they have a greater number of connections. Because ideas with more connections are most easily recalled, this leads to greater recall of central than of peripheral information. The fact that poor readers display a centrality effect suggests that they connect the text's ideas to some extent; however, their centrality deficit implies that they fail to connect the text's ideas to the same degree typical readers do.

The purpose of the present study was to examine the recall of another group of readers faced with the task of comprehending with limited cognitive resources: foreign language (L2) learners. Just as poor readers must allot a large proportion of cognitive resources to lower level processes (e.g., word decoding) and are left with relatively few resources remaining for higher level comprehension processes, L2 learners face a similar challenge. L2 comprehension results in a greater cognitive workload than does first language (L1) comprehension (Hasegawa, Carpenter, \& Just, 2002) because L2 learners must devote more cognitive resources to lower level processes, such as word identification, semantic access, and syntactic processing and, as a result, fewer cognitive resources remain for higher level processes, such as forming connections among a text's related ideas. Examining the memory for text of L2 learners can provide further insight into the centrality deficit. Previous work has demonstrated that L2 learners of all skill levels generally show a centrality effect, recalling more central than peripheral ideas (Barry \& Lazarte, 1995; Lund, 1991; Schmidt-Rinehart, 1994). But if we found that L2 learners, like poor readers, also display a centrality deficit, it would provide further support for the idea that the underlying cause of the centrality deficit may be comprehending with limited cognitive resources.

In this study, we examined readers' memory for text when reading in their L2 (Spanish), as compared with their L1 (English). The purpose was not just to examine how reading in L2 would affect the amount recalled, but to determine whether L2 reading would affect the quality of the recall-which specific ideas tended to be remembered. We examined recall of central and peripheral information after readers read L2 versus L1 passages to determine whether reading in L2 would differentially affect the quantity of ideas recalled-specifically, whether it would impact central information more than peripheral information or vice versa - or whether all ideas would be equally depressed by the reduced cognitive resources associated with reading in L2. We also examined whether any observed deficits in L2 discourse comprehension could be overcome when the reader had prior knowledge (PK) of the passage topic. PK is known to improve text comprehension in general (e.g., Haenggi \& Perfetti, 1992; Rawson \& Kintsch, 2004; Samuelstuen \& Bråten, 2005), but our aim was to determine how PK of the passage topic would specifically influence recall of central and peripheral information.

\section{Prior knowledge}

Reading comprehension is a constructive process that involves integrating the words in the text with one's knowledge of the world, so it makes sense that having PK of the passage topic facilitates comprehension (e.g., Haenggi \& Perfetti, 1992; Rawson \& Kintsch, 2004; 
Samuelstuen \& Braten, 2005). However, Miller and Keenan (2009) found that PK even allows poor readers to compensate for their centrality deficit. Poor readers showed a centrality deficit, relative to good readers, when neither group had PK of the passage topic; but when both groups had PK of the topic, poor readers no longer showed the deficit (see the bottom half of Fig. 1). PK did not affect the performance of the good readers, however, suggesting that they were able to form connections among the text's ideas on the basis of text cues alone.

No study has examined the effect of PK on the quality of recall in L2, but a number of studies have clearly demonstrated that PK increases the quantity of L2 recall, facilitates L2 question answering, and allows readers to ignore irrelevant information (Barry \& Lazarte, 1995; Carrell, 1983; Schmidt-Rinehart, 1994; Taglieber, Johnson, \& Yarbrough, 1988; Tudor, 1988; Tyler, 2001). The present study assessed readers' memory for text to determine whether they would show a centrality deficit when reading L2 passages, relative to L1 passages, and if they did, whether PK of the passage topic would help the readers compensate for such a deficit.

Because PK and IQ are typically correlated, and because either could influence memory for text, we assessed PK as a within-participants variable. In this way, general intelligence was controlled across the levels of PK, so that we avoided this confound and could attribute any knowledge effects to knowledge about the topic, rather than to general intelligence.

\section{Language proficiency}

This study also examined whether L2 proficiency would differentially impact the proportion of central and peripheral ideas recalled. L2 proficiency is a clear predictor of L2 comprehension when comprehension is measured by openended questions (e.g., Asfaha, Beckman, Kurvers, \& Kroon, 2009), cloze procedure (e.g., Gottardo \& Mueller, 2009), and quantity of the ideas recalled (Fecteau, 1999), so we expected that when low-proficiency L2 readers were reading L2 passages, they would also show a more striking centrality deficit than would high-proficiency L2 readers. The basis for this prediction was that if the centrality deficit is a by-product of L2 learners having to devote more cognitive resources to lower level processes, the centrality deficit should be most apparent when a person has lower proficiency in their L2. We also examined whether PK interacts with proficiency to influence L2 readers' text representations. We expected that PK would provide greater compensation for the individuals who needed it most-those with low L2 proficiency.
It is important to note that we made a specific effort in this study to distinguish between L2 proficiency and general verbal ability. We advanced previous work on L2 proficiency by having assessments of both the participants' L1 ability and their L2 ability. By comparing the low and high L2 proficiency readers on their L1 ability, we could determine whether any results we obtained for proficiency reflected the effects of L2 proficiency per se or whether they reflected differences in general verbal ability and, thus, would be evident in differences between the groups for L1 as well. Because we hypothesized that the centrality deficit is a function of proficiency in the participants' L2, we expected that high- and low-proficiency L2 readers would differ only on L2 ability, and not also on L1, although we recognized that general verbal ability and L2 proficiency could be correlated.

\section{Overview}

The goal of this study was to better understand the centrality deficit, which is believed to stem from a reader's inability to form sufficient connections among the text ideas that allow centrality to fully emerge (Miller \& Keenan, 2009). The present study assessed whether participants showed a greater deficit in the recall of central than of peripheral ideas (i.e., a centrality deficit) when participants recalled passages that they read in their L2, as compared with those they read in their L1. Participants were native English-speaking undergraduates learning Spanish as an L2. Participants read both L1 passages and L2 passages; thus, language was a within-participants variable. The importance of the idea units in each passage were rated by an independent group of undergraduates, and these ratings were used to determine which ideas were central and which were peripheral. The proportions of central and peripheral information recalled by the participants reading in their L1 and L2 were compared.

Because participants did, in fact, show a centrality deficit when reading L2, as compared with L1, passages, we then asked whether having greater Spanish proficiency reduces the centrality deficit. Spanish proficiency was a between-participants variable (high or low), based on participants' performance on a Spanish exam, and it was shown to not be the same as general verbal ability, because the high and low L2 proficiency groups did not differ on the measure of L1 verbal ability, the Woodcock-Johnson Letter-Word Identification test. We also examined whether having PK of the passage topic can help compensate for the centrality deficit. PK was a within-participants variable; a variety of passage topics were included, so that each participant had high PK of some topics and low PK of others. 


\section{Method}

Participants

Forty-three undergraduates previously or currently enrolled in their university's Spanish curriculum participated in the study and received monetary compensation or, if they preferred, extra credit for a psychology course. All the participants were native English speakers who were recruited from beginning or intermediate Spanish courses. Two additional participants were tested but were excluded because they were not native English speakers. Data were lost from 1 participant's recall on one passage (Pele) because the audio recorder failed to record the passage, but data from this participant's remaining nine passages were included in the analyses. Also, 1 participant does not have a score for the Woodcock-Johnson Letter-Word Identification test, due to administration error, but the rest of this participant's data were included in the analyses.

\section{Design}

Every participant read a total of ten passages: eight L2 Spanish passages and two L1 English passages. The reason that fewer passages were given in English than in Spanish has to do with the PK variable. We anticipated that PK would play a compensatory role and, thus, would influence memory only for text of L2 passages. Participants read a greater number of L2 Spanish passages in order to increase the likelihood of scoring within the high-PK category on some of the L2 passages and within the low-PK category on others (see Table 1 for a breakdown of participants' knowledge of each passage topic). Because we did not evaluate the effect of PK on the L1 passages, we administered only two L1 passages. Averaging across these two passages (as opposed to using only one passage) decreased the influence of passage variability but did not

Table 1 Number of participants reading each passage in Spanish with low prior knowledge (PK) and with high PK

\begin{tabular}{lll}
\hline Passage & Spanish Low PK & Spanish High PK \\
\hline Battle of the Alamo & 14 & 20 \\
Ferdinand Magellan & 13 & 21 \\
Hannah Montana & 6 & 29 \\
Galapagos Islands & 13 & 21 \\
Manatees & 8 & 27 \\
Margaret Mead & 34 & 0 \\
Panama Canal & 8 & 26 \\
Pele & 14 & 19 \\
Stegosaurus & 19 & 16 \\
Tower of London & 24 & 11 \\
\hline
\end{tabular}
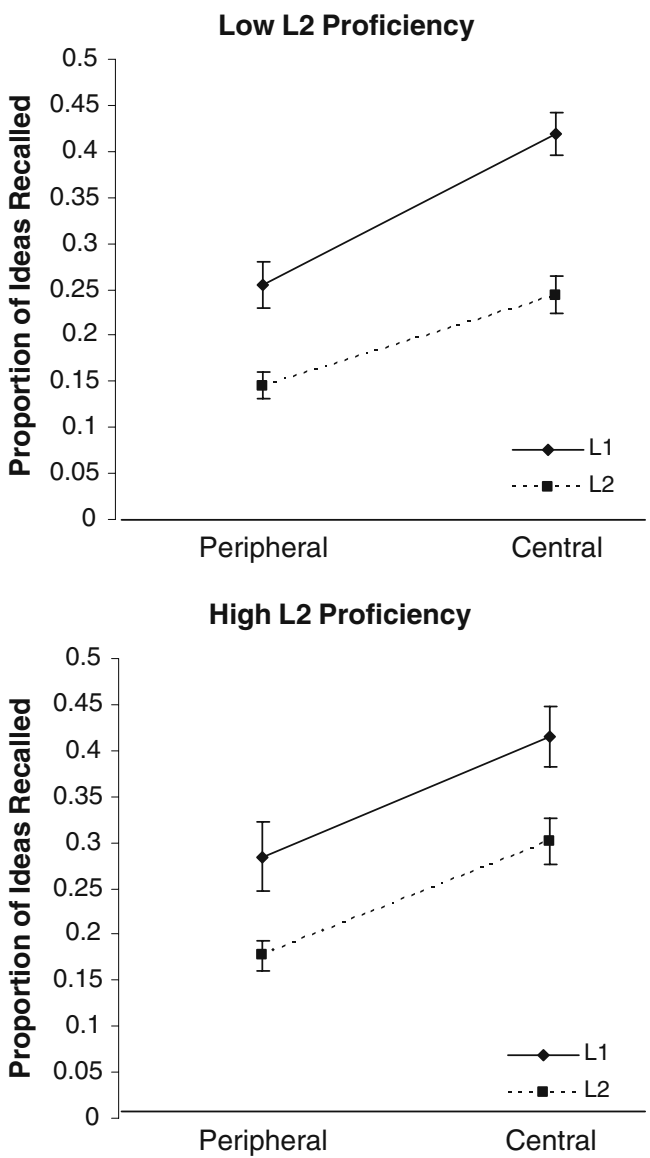

Fig. 2 Proportions of central and peripheral ideas recalled by participants with either low L2 proficiency or high L2 proficiency, reading in their L1 and L2

substantially increase the length of the testing session. Assignment of passages to language version (English or Spanish) for a particular participant was counterbalanced across participants. Each participant read every passage once, either the English or the Spanish version. Passage order was randomized across participants; each participant had a different passage order.

\section{Materials}

Passages All the passages were expository, 257-395 words in length, and at the 5 th-7th grade reading level as determined by the Flesch-Kincaid grade-level rating system. All ten passages were translated into Spanish by two bilingual individuals - one Spanish-English and one English-Spanish. They translated the passages back and forth between the two languages until they agreed that the English and Spanish passages were equivalent, paying careful attention that the translations did not change the passages' meaning, length, or overall reading difficulty. The English and Spanish versions were entered into languagespecific readability formulas (English, Flesch Reading 
Table 2 Descriptive statistics of participants with low and high Spanish proficiency. Means and standard deviations are presented

\begin{tabular}{lllll}
\hline & $n$ & Spanish Exam & WJ & ACT \\
\hline Low proficiency & 16 & $17.00(2.61)$ & $70.33(3.13)$ & $26.33(3.04)$ \\
High proficiency & 18 & $28.67(2.87)$ & $69.94(3.72)$ & $27.53(3.91)$ \\
\hline
\end{tabular}

Ease; Spanish:Huerta Reading Ease) that provide readability estimates using a 0-100 scale, with lower scores indicating greater reading difficulty. The English $(M=$ 73.07) and Spanish $(M=76.02)$ readability estimates did not significantly differ, $t(9)=1.64, p>.05$.

Classifying ideas as central versus peripheral Each passage was broken down into idea units based on clause structure. Sentences were parsed by two individuals independently, and disagreements were settled by a third individual.

A variety of techniques have been employed to determine which ideas in a passage are central and which are peripheral. Some, such as propositional hierarchies (Kintsch, 1974; Kintsch \& Keenan, 1973) and causal networks (van den Broek \& Trabasso, 1986), can be laborious to apply to long passages such as those used in the present study. A measure that correlates highly with more theoretical assessments of centrality is ratings of importance (Albrecht \& O'Brien, 1991; O'Brien \& Myers, 1987), which is the method we used to define the centrality of the idea units in our passages.

A norming study was performed to determine whether each idea unit in the ten passages was central or peripheral to the passage's overall meaning. Native English-speaking undergraduates rated the importance of each item on the idea checklist, using a Likert scale ranging from 0 (the idea unit is unimportant to the overall meaning of the story) to 7 (the idea unit is very important to the overall meaning of the story). Each participant provided ratings for six passages in a 1-hr testing session. Each passage was rated by a total of 20 undergraduates. Mean ratings were calculated, and the idea units whose mean ratings fell above the median were considered central, while those below the median were considered peripheral. We also defined centrality using just the ideas in the highest quartile as central and those in the lowest quartile as peripheral. The two methodologies yielded the same pattern of overall results, so we confine the presentation of the results to just the median split method, which is consistent with the method used by Miller and Keenan (2009).

Prior knowledge assessment Participants' PK of the passage topics was assessed by a test prior to reading the passages. Participants were asked one open-ended question about each of the ten topics, such as "Who was Pele?" If the participant gave a correct response, their response was queried in order to assess the extent of their knowledge, such as "Tell me everything you know about Pele." Participants were encouraged to state everything they knew about the topic. Participants' responses were scored by two independent raters (Cronbach's $\alpha=.98$ ) and were categorized as low $\mathrm{PK}$ or high $\mathrm{PK}$ for each passage. If participants did not have any knowledge of the topic, they were included in the low-PK group. By limiting the number of questions about each topic and administering them at the very beginning of the testing session, rather than directly before their corresponding passages, we hoped to reduce the potential effects that artificial knowledge activation might have on memory for text.

Because the effects of PK were assessed within participants, participants who either had high PK about every passage topic or who had low PK about every passage topic were excluded from the PK analyses. One participant's data were excluded from the PK analyses for this reason. Likewise, if a participant scored in a given PK group on only one passage, this participant's data were also excluded, because it was thought that at least two passages needed to be averaged to have a stable measure of how recall was impacted by knowledge. Seven participants' data were excluded from the PK analyses for this reason.

Assessment of English (L1) word identification skills The Letter-Word Identification subtest of the Woodcock-Johnson Tests of Achievement-III (Woodcock, McGrew, \& Mather, 2001) was used to measure English word reading ability to determine whether the high and low L2 proficiency groups differed in their L1 verbal ability. Participants also selfreported their ACT scores. These two L1 measures allowed us to determine the specificity of any L2 proficiency effects, whether it was specifically L2 proficiency or more general language ability.

Spanish (L2) proficiency assessment An exam consisting of 38 multiple-choice questions selected from an online Spanish quiz (Fun Spanish Quizzes, n.d.) assessed participants' knowledge of Spanish grammar and vocabulary and targeted beginning, intermediate, and advanced proficiency levels. Raw scores ranged from 12 to 35 correct $(M=$ 23.19, $S D=5.79)$. Because we were interested in understanding how proficiency interacts with memory for text and prior knowledge, we divided our sample into high 
and low Spanish proficiency groups. Those below the 33rd percentile composed the low-proficiency group $(n=16)$, and those above the 66th percentile composed the highproficiency group $(n=18)$.

\section{Procedure}

Participants were first given the PK exam in order to assess their knowledge of the passage topics (5-10 min). The PK exam was asked and answered orally in English. No time limit was enforced. Responses were recorded and subsequently independently scored by two raters. After the PK assessment, the Woodcock-Johnson Letter-Word Identification test was administered (5 $\mathrm{min})$, followed by the Spanish proficiency exam (10-15 min). Participants read the questions silently and marked their answers on the paper provided.

After these preliminary exams, participants read the first passage and immediately recalled everything they could remember from the passage. All the participants read silently in English or Spanish and then freely recalled each passage in English, regardless of the language in which it was read. Lee (1986) showed that participants recalled significantly more ideas when they recalled L2 passages in their L1 rather than in their L2; therefore, as was suggested by Lee and others (Donin \& Silva, 1993; Wolff, 1987), participants in this study recalled the passages in English, so that their memory for text was not masked by limited Spanish production skills. The reason they were asked to read silently is that L2 readers exhibit better comprehension when reading silently than when reading aloud (Bernhardt, 1983). Bernhardt (1983) suggested that reading silently allows L2 readers to focus cognitive resources on comprehension, rather than on pronunciation.

This procedure was repeated for the remaining nine passages. Reading and recalling the ten passages required approximately $60 \mathrm{~min}$. Recalls were digitally recorded, subsequently transcribed, and scored using the idea checklists. A subset of the passages $(n=80)$ was scored by two raters, and interrater reliability was very high (Cronbach's $\alpha$ $=.98$ ).

\section{Results}

Passage validity

As was previously stated in the introduction, a wellestablished finding in the text memory literature is that individuals recall a greater number of central than of peripheral ideas from a passage (Britton et al., 1980; Brown \& Smiley, 1978; Cirilo \& Foss, 1980; Curran et al., 1996; Keenan \& Brown, 1984; Kintsch \& Keenan, 1973; Kintsch et al., 1975; Kintsch \& van Dijk, 1978; Meyer, 1974; Miller \& Keenan, 2009). We first wanted to ensure that all of our passages met this expectation. If central ideas do not emerge from a given passage as being central, it is misleading to compare the proportions of central and peripheral ideas recalled from this passage with those from other passages. Depending on the comparison, it could over- or underestimate the existence of a centrality deficit and make it difficult to draw conclusions about the coherence of the L2 text representation.

To test whether the passages showed a centrality effect, a repeated measures ANOVA was performed on each passage, comparing the proportion of central and peripheral information recalled. Participants (L1 and L2 combined) recalled significantly more central than peripheral ideas from eight of the ten passages (see Table 3). The two passages from which participants did not recall more central than peripheral information were Hannah Montana and Battle of the Alamo. These passages were not included in subsequent analyses because their atypical centrality patterns would not allow us to assess our main question of how L2 processing impacts the centrality of readers' recall.

\section{Centrality deficit}

The mean proportions of central and peripheral ideas recalled by participants reading in their L1 and L2 were examined using a $2 \times 2$ repeated measures ANOVA, with language (L1, L2) and centrality of recalled idea units (central, peripheral) as within-participants variables and proportion of idea units recalled as the dependent variable. As was expected on the basis of previous research, there was a main effect of language, $F(1,40)=96.84, p<.001$, partial $\eta^{2}=.71$, such that participants recalled a greater proportion of ideas when reading in their L1, as compared

Table 3 Mean proportions of peripheral and central ideas recalled from each passage, averaged over L1 and L2 (and standard deviations)

\begin{tabular}{lll}
\hline Passage & Peripheral & Central \\
\hline Battle of the Alamo & $.25(.12)$ & $.25(.13)$ \\
Ferdinand Magellan & $.24(.13)$ & $.30(.15)^{*}$ \\
Hannah Montana & $.41(.15)$ & $.42(.14)$ \\
Galapagos Islands & $.22(.14)$ & $.33(.11)^{*}$ \\
Manatees & $.11(.11)$ & $.25(.13)^{*}$ \\
Margaret Mead & $.22(.13)$ & $.34(.15)^{*}$ \\
Panama Canal & $.17(.11)$ & $.37(.15)^{*}$ \\
Pele & $.27(.12)$ & $.34(.11)^{*}$ \\
Stegosaurus & $.13(.09)$ & $.27(.18)^{*}$ \\
Tower of London & $.16(.12)$ & $.31(.11)^{*}$ \\
\hline
\end{tabular}

*Significantly more central than peripheral ideas recalled, $p<.001$ 
with their L2. There was also a main effect of centrality, $F$ $(1,40)=192.75, p<.001$, partial $\eta^{2}=.83$, with participants recalling significantly more central than peripheral information. Tests of simple effects showed that the centrality effect was highly significant for participants reading $\mathrm{L} 1, F(1,40)=$ $84.85, p<.001$, partial $\eta^{2}=.68$, and L2, $F(1,40)=176.15$, $p<.001$, partial $\eta^{2}=.82$ passages.

Our main question of how reading in a foreign language impacts the construction of a mental representation of a text was addressed by examining the interaction of language and centrality. Participants showed a significantly greater deficit in the recall of central than of peripheral information when reading L2 passages than when reading L1 passages, $F(1,40)=4.79, p<.05$, partial $\eta^{2}=.11$. Thus, when reading in $\mathrm{L} 2$, readers show a centrality deficit, relative to their $\mathrm{L} 1$ recall.

If the deficit occurs because reading in L2 diminishes the cognitive resources available to connect ideas together, we should find that participants with lower L2 proficiency show more of a centrality deficit than do those with higher proficiency. This prediction was tested with a mixed design ANOVA with centrality (peripheral, central) and language (L1, L2) as within-participants variables and proficiency (low, high) as a between-participants variable. As Fig. 2 shows, the pattern of findings supports our prediction that the centrality deficit should be greater when language proficiency is lower. The top panel of Fig. 2 shows the participants with low Spanish proficiency. Planned comparisons revealed that they showed a significant centrality deficit when reading L2 passages, as compared with L1 passages [centrality $\times$ language interaction: $F(1,15)=7.29$, $p<.05$, partial $\eta^{2}=.33$ ]. The bottom panel of Fig. 2 shows the high Spanish proficiency group; here, the centrality deficit is no longer evident [centrality $\times$ language interaction: $F(1,15)<1$, partial $\left.\eta^{2}=.002\right]$. Although the pattern of findings supports the notion that recall of central information is differentially affected by language proficiency, it should be noted that the three-way interaction between centrality (peripheral, central), language (L1, L2), and proficiency (low, high) was not significant, $F(1,30)=$ 2.38, $p>.05$, partial $\eta^{2}=.07$.

\section{Specificity of L2 proficiency}

The low and high L2 proficiency groups were compared on their English verbal skills, as measured by the WoodcockJohnson Letter-Word Identification test and their selfreported ACT scores. As can be seen in Table 2, the L2 proficiency groups did not differ in their L1 verbal ability as measured by either the Woodcock-Johnson Letter-Word Identification test, $F(1,31)<1$, or ACT, $F(1,22)<1$. To further test this claim, we compared the low- and highproficiency L2 readers on their recall of L1 passages in a 2 (central, peripheral) $\times 2$ (low proficiency, high proficiency) ANOVA. There was no main effect of L2 proficiency on recall of $\mathrm{L} 1$ passages, $F(1,30)<1$, nor was the interaction between centrality and proficiency, $F(1,30)<1$, significant when L1 passages were recalled.

\section{Knowledge compensation}

After finding that participants showed a centrality deficit when reading $\mathrm{L} 2$, as compared with $\mathrm{L} 1$, passages, particularly when they are less proficient in their L2, we next assessed whether having PK could help compensate for this deficit. In order to analyze the PK effects, we standardized recall within each passage and then calculated the average $z$-score for each participant's high-PK passages and the average $z$-score for their low-PK passages. This standardization was necessary because the distribution of PK on several passages was unbalanced (see Table 1) and some passages were more memorable than others (i.e., associated with relatively high or low recall proportions; see Table 3). If recall data were not standardized, the combination of these two issues could disproportionately impact the results. For example, the majority of participants had high PK of manatees and, as compared with other passages, recalled a relatively low proportion of ideas from the Manatees passage. The combination of these two issues would unduly lower the mean proportion of ideas recalled by the high-PK group if the recall data were not standardized.

We next assessed how the size of the centrality deficit in L2, relative to L1, depends on L2 proficiency and PK of the passage topic. Participants read passages that each fell into one of three categories: L2 passages about which they had high PK, L2 passages about which they had low PK, and L1 passages. A passage (L2 passages/high PK, L2 passages/low PK, L1 passages) $\times$ proficiency (high L2 proficiency, low L2 proficiency) $\times$ centrality (central, peripheral) mixed design ANOVA revealed that PK indeed operated as a compensatory tool for the low-proficiency L2 readers. When low-proficiency L2 readers read passages with high PK, their centrality deficit was attenuated, but when they read passages with low PK, they continued to demonstrate the deficit. This finding is evidenced by a significant three-way passage $\times$ proficiency $\times$ centrality interaction, $F(2,19)=5.04, p<.05$, partial $\eta^{2}=.35$ (see Fig. 3).

Planned comparisons revealed that low L2 proficiency readers showed a significant centrality deficit when reading L2, as compared with L1, passages when they had low PK of the L2 passage [centrality $\times$ passage interaction: $F(1,12)$ $=7.56, p<.05$, partial $\eta^{2}=.39$ ], but when these same readers had high PK of the L2 passage, they no longer showed a centrality deficit, as compared with their L1 recall [centrality $\times$ passage interaction: $F(1,12)=1.14, p>.05$, 

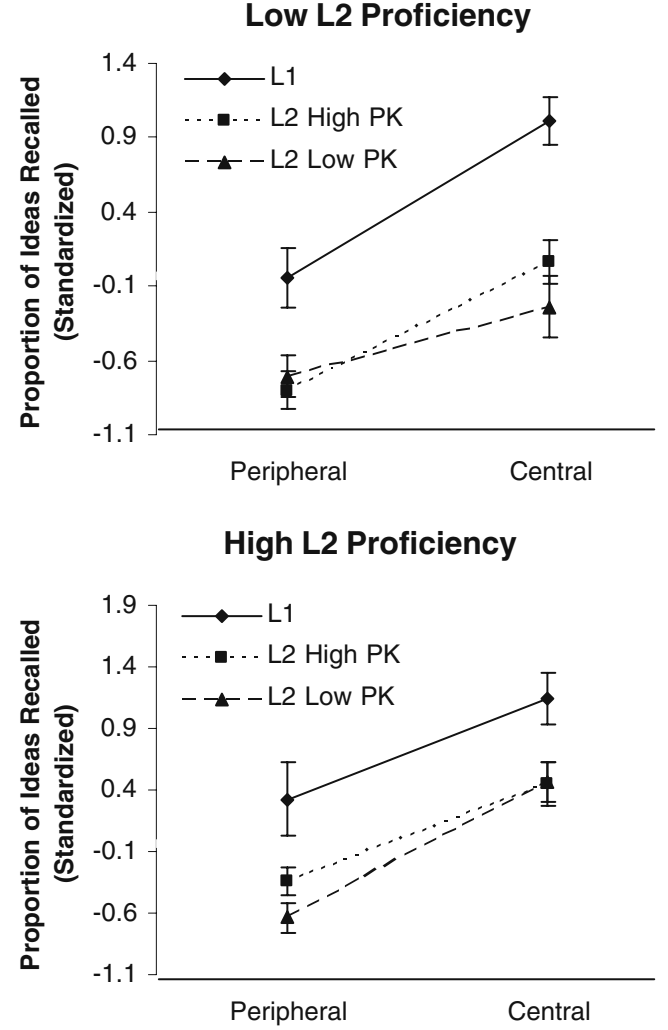

Fig. 3 Proportions of central and peripheral ideas recalled by participants with either low L2 proficiency or high L2 proficiency, reading in their L1 and in their L2 with high and low prior knowledge (PK) of the passage topic. Proportions were standardized within each passage

partial $\left.\eta^{2}=.09\right]$. However, this pattern changes for highproficiency L2 readers. High-proficiency L2 readers did not show a centrality deficit when reading L2, as compared with L1, passages, regardless of whether they were reading L2 passages of which they had low PK or high PK [centrality $\times$ passage interaction: $F(1,8)=1.21, p>.05$, partial $\eta^{2}=.13$, and $F(1,8)<1$, respectively). In sum, both high PK and high L2 proficiency can help L2 readers build the appropriate connections among the text's ideas and, thereby, compensate for the centrality deficit that they show when reading $\mathrm{L} 2$, relative to $\mathrm{L} 1$, passages.

A second set of planned comparisons assessed whether participants reading L2 passages showed a centrality deficit in their recall of low-PK L2 passages, relative to their recall of high-PK L2 passages. We included these planned comparisons to test whether it was the L1 recall that was pulling the interaction demonstrated in the previous set of planned comparisons. A 2 (high PK, low PK) $\times 2$ (high proficiency, low proficiency) $\times 2$ (central, peripheral) mixed design ANOVA resulted in a significant three-way interaction, $F(1,21)=8.64, p<.01$, partial $\eta^{2}=.29$ (see Fig. 3). Again, the low-proficiency L2 readers showed a significant centrality deficit when they had low PK of L2 passages, as compared with when they had high PK of L2 passages $[\mathrm{PK} \times$ centrality interaction: $F(1,12)=5.83, p<$ .05 , partial $\left.\eta^{2}=.33\right]$. High-proficiency L2 readers, however, did not show a centrality deficit when reading L2 passages for which they had low PK, as compared with L2 passages for which they had high PK.

It should be noted that participants still recalled fewer ideas overall in their L2, relative to their L1, regardless of whether they possessed PK and/or high L2 proficiency. This is evidenced by a significant main effect of language among high L2 proficiency readers with low PK, $F(1,8)=$ $13.82, p<.01$, partial $\eta^{2}=.63$, high L2 proficiency readers with high PK, $F(1,8)=22.52, p<.01$, partial $\eta^{2}=.74$, low proficiency readers with low PK, $F(1,12)=37.02, p<$ .001 , partial $\eta^{2}=.76$, and low L2 proficiency readers with high PK, $F(1,12)=26.63, p<.001$, partial $\eta^{2}=.69$.

\section{Discussion}

The goal of this study was to gain insight into comprehension processes and individual differences in these processes by examining whether a centrality deficit occurs in memory for text when L2 learners are reading in their L2 versus their L1. Using language as a within-participants variable, we compared the proportions of central and peripheral ideas recalled when participants read in L2 versus L1. The main finding was that when reading in L2, participants showed a greater deficit, relative to their $\mathrm{L} 1$, in recalling central information - a centrality deficit - than in recalling peripheral information. This study is the first to demonstrate the specificity of the effects of L2 on the nature of the text representation.

Although previous studies have recognized that L2 readers display a centrality effect (Barry \& Lazarte, 1995; Lund, 1991; Schmidt-Rinehart, 1994), none have compared the slope of this centrality effect with that of the participants reading in their L1. As in previous studies, we too found that participants reading in their L2 recalled more central than peripheral ideas overall. However, of primary significance was our finding that participants showed their greatest deficit when reading in their L2, as compared with their L1, for central information. Although showing their greatest deficit for the ideas they recall the best is counterintuitive, the centrality deficit can be explained by realizing that it is an indication of a failure to build a coherent representation of the text. Central ideas emerge as central because they have a greater number of connections. The ideas with more connections are most easily recalled, and this leads to greater recall of central than of peripheral information. The fact that L2 readers display a centrality effect suggests that they connect the text's ideas to some extent; however, their centrality deficit 
implies that they fail to connect the text's ideas to the same degree that $\mathrm{L} 1$ readers do.

We theorized that this centrality deficit stems from the fact that L2 reading comprehension requires that a greater proportion of cognitive resources be devoted to lower level processes (e.g., word identification or understanding syntactic structure) than $\mathrm{L} 1$ reading does. Because participants reading in their L2 must allocate more resources to lower level processes, fewer resources remain for higher level processes, including forming connections among the text's ideas, and consequently, less differentiation between central and peripheral ideas occurs.

This theory is supported by the finding that the centrality deficit is closely tied to L2 proficiency. When participants were divided according to their level of L2 proficiency (i.e., low proficiency vs. high proficiency), the centrality deficit was apparent in the memory for text of the readers with low L2 proficiency, but not in those with higher L2 proficiency. Readers with low L2 proficiency must devote even more cognitive resources to lower level processes than do those with higher L2 proficiency and, consequently, are left with even fewer resources to form connections among the text's ideas. It is important to note that readers with low L2 proficiency did not differ from those with high L2 proficiency on L1 verbal ability or in their memory for text of L1 passages, which strongly suggests that it was lower L2 proficiency that caused the centrality deficit, rather than differences between the two proficiency groups in general verbal ability.

In addition to being the first study to recognize that reading in L2 impacts the derivation of what is most central and to offer a theory to explain this centrality deficit, another major contribution of this study was to identify a compensatory mechanism that can help alleviate the centrality deficit: PK of the passage topic. Using a design where PK was a within-participants variable, we compared memory for L2 passages when participants did not have PK of the passage topic with memory for those when they did. PK served as a compensatory mechanism for low L2 proficiency participants. They showed a centrality deficit when recalling passages for which they had low PK, as compared with those for which they had high PK. This interaction indicates that when a reader with low L2 proficiency is aided by $\mathrm{PK}$, this knowledge not only facilitates the overall amount recalled, but also improves recall of the central ideas in particular. It is important to note that because PK was a within-participants variable, the differences we obtained for different levels of knowledge are not confounded by participant differences in cognitive abilities, as they are when knowledge is treated as a between-participants variable, as have most previous studies of topic knowledge (e.g., Recht \& Leslie, 1988; Samuelstuen \& Braten, 2005). Because we compared the same person's recall when they did and did not have knowledge of the passage topic, we can conclude that it is a difference in PK of the specific passage topic that alleviates the centrality deficit, rather than a variable related to general cognitive ability or degree of L2 proficiency.

When we compared participants' recalls of L2 passages with and without PK with their recall of L1 passages to determine whether the effect of PK was sufficient to compensate for the degree of centrality deficit shown by low-proficiency L2 readers, as compared with L1 readers, we found out just how powerful PK can be. Low L2 proficiency readers, when they were not aided by PK, displayed a centrality deficit when reading in their L2, as compared with their L1. Remarkably, however, when those same participants were equipped with PK of the passage topic, the centrality deficit disappeared. Thus, prior topic knowledge is a powerful variable that allows centrality to emerge to the same degree for low-proficiency readers when reading L2 texts as when reading $\mathrm{L} 1$ texts.

This finding parallels that of Miller and Keenan (2009), who found that possessing PK of the passage topic helped poor readers resolve the centrality deficit. They theorized that readers who have difficulties using text information to establish connections can offset those difficulties by using their PK in a number of ways to form connections. They proposed that PK might provide the reader with a preexisting idea of what is central (Goetz, Schallert, Reynolds, \& Radin, 1983). They also proposed that PK could decrease the amount of resources required to form connections among text ideas, because those connections are already available. Finally, PK may enable the reader to shift their cognitive resources from lower level processes toward higher level comprehension processes.

In short, these findings suggest that as long as individuals reading L2 passages are equipped with either PK of the passage topic or a higher level of L2 proficiency, they will no longer demonstrate the centrality deficit, as compared with their L1 reading performance. Only individuals with lower L2 proficiency who do not possess PK of the passage topic are impacted. Both of these compensation tools are theorized to operate in a similar mannerby facilitating the formation of connections among the text's ideas, which in turn allows central ideas to be recalled more easily.

In addition to helping us understand the centrality deficit, this study also provides insight into how L2 comprehension should be assessed. Most studies that have assessed L2 discourse comprehension have done so with multiple-choice comprehension questions (e.g., Brantmeier, 2005; Wolf, 1993). While such questions have the obvious advantage that they are easy to score, they typically provide limited insight into the coherence of an individual's text representation. Rather than assessing the reader's compre- 
hension of the entire text, multiple-choice questions target specific ideas. Furthermore, they often provide clues about the correct answer (Bernhardt, 1991), are susceptible to guessing strategies (Wolf, 1993), and often include items that are passage independent (Coleman, Lindstrom, Nelson, Lindstrom, \& Gregg, 2010; Keenan \& Betjemann, 2006). Good performance does not necessarily mean that the reader has formed the appropriate connections and built a coherent representation of the text. This study suggests that a better way to assess comprehension is to ask the reader to retell what he or she has just read and examine his or her memory for the text, because then one can determine whether the reader formed appropriate connections among the text's ideas, allowing the most central ideas to be retained. Examining memory for text assesses comprehension of the entire passage rather than specific ideas and, thus, is a preferable method for assessment when the goal is to examine the reader's representation of the overall meaning of a text.

The insights provided by this study into the mechanisms of discourse comprehension suggest possible avenues for future research. Taken together, the results of the present study and Miller and Keenan (2009) suggest that the centrality deficit results from comprehending with limited resources. Future work should further attempt to isolate this mechanism by implementing a more direct measure of the amount of resources available for comprehension. Second, because we have shown that reading in L2 makes it more difficult to derive the most central ideas, it would be interesting to explore whether L2 readers can be aided by highlighting the central information in various ways. Studies could also explore whether training L2 readers to use metacognitive techniques requiring them to focus more on central ideas would be an effective instruction strategy. Also, because the present findings show that PK can serve as a powerful compensatory mechanism, future work could explore the relative benefits of improving the reader's topic knowledge before engaging in L2 reading. Finally, our finding that PK compensates for the centrality deficit suggests that L2 comprehension assessment should take into account the knowledge that the reader brings to the table. In other words, it is important to be aware of the reader's PK (or lack thereof) and not have L2 comprehension assessment occur in a vacuum.

Acknowledgment We thank Anh Hua, Dr. Iris Mauss, Chelsea Meenan, Dr. Bruce Pennington, and Sarah Priebe for discussions of the data. We also thank all the participants and the faculty of the University of Denver Spanish Department who enabled participant recruitment.

Author Note Amanda C. Miller and Janice M. Keenan, Department of Psychology, University of Denver. This project was funded by a grant to Amanda Miller from the Educational Testing Services, Princeton, NJ, for Doctoral Research in Second or Foreign Language
Assessment. Janice M. Keenan was supported by a grant from the National Institutes of Health, P50 HD27802.

Correspondence concerning this article should be sent to Amanda Miller or Jan Keenan, Department of Psychology, University of Denver, Denver, CO80208. E-mail: miller23@gmail.com or jkeenan@du.edu.

\section{References}

Albrecht, J., \& O'Brien, E. (1991). Effects of centrality on retrieval of text-based concepts. Journal of Experimental Psychology: Learning, Memory, and Cognition, 17, 932-939. doi:10.1037/ 0278-7393.17.5.932

Asfaha, Y., Beckman, D., Kurvers, J., \& Kroon, S. (2009). L2 reading in multilingual Eritrea: The influences of L1 reading and English proficiency. Journal of Research in Reading, 32, 351-365. doi:10.1111/j.1467-9817.2009.01399.x

Barry, S., \& Lazarte, A. (1995). Embedded clause effects on recall: Does high prior knowledge of content domain overcome syntactic complexity in students of Spanish? Modern Language Journal, 79, 491-504. doi:10.2307/330001

Bernhardt, E. B. (1983). Three approaches to reading comprehension in intermediate German. Modern Language Journal, 67, 111115. doi: $10.2307 / 328281$

Bernhardt, E. B. (1991). Reading development in a second language: Theoretical, empirical, and classroom perspectives. Norwood: Ablex.

Brantmeier, C. (2005). Effects of reader's knowledge, text type, and test type on L1 and L2 reading comprehension in Spanish. Modern Language Journal, 89, 37-53. doi:10.1111/j.00267902.2005.00264.x

Britton, B., Meyer, B., Hodge, M., \& Glynn, S. (1980). Effects of the organization of text on memory: Tests of retrieval and response criterion hypotheses. Journal of Experimental Psychology: Human Learning and Memory, 6, 620-629. doi:10.1037/0278-7393.6.5.620

Brown, A., \& Smiley, S. (1978). The development of strategies for studying texts. Child Development, 49, 1076-1088. doi:10.2307/ 1128747

Carrell, P. L. (1983). Three components of background knowledge in reading comprehension. Language Learning, 33, 183-207.

Cirilo, R., \& Foss, D. (1980). Text structure and reading time for sentences. Journal of Verbal Learning and Verbal Behavior, 19, 96-109. doi:10.1016/S0022-5371(80)90560-5

Coleman, C., Lindstrom, J. H., Nelson, J. M., Lindstrom, W., \& Gregg, N. (2010). Passageless comprehension on the NelsonDenny reading test: Well above chance for university students. Journal of Learning Disabilities, 43, 244-249. doi:10.1177/ 0022219409345017

Curran, C., Kintsch, E., \& Hedberg, N. (1996). Learning-disabled adolescents' comprehension of naturalistic narratives. Journal of Educational Psychology, 88, 494-507. doi:10.1037/00220663.88.3.494

Donin, J., \& Silva, M. (1993). The relationship between first- and second-language reading comprehension of occupation-specific texts. Language Learning, 43, 373-401. doi:10.1111/j.14671770.1993.tb00619.x

Fecteau, M. (1999). First- and second- language reading comprehension of literary texts. Modern Language Journal, 83, 475-493. doi:10.1111/0026-7902.00036

Fun Spanish Quizzes, 123TeachMe. (n.d.) Retrieved April 2008, from http://www.123teachme.com/learn spanish/online spanish test.

Goetz, E. T., Schallert, D. L., Reynolds, R. E., \& Radin, D. I. (1983). Reading in perspective: What real cops and pretend burglars look for in a story. Journal of Educational Psychology, 75, 500-510. 
Gottardo, A., \& Mueller, J. (2009). Are first- and second-language factors related in predicting second-language reading comprehension? A study of Spanish-speaking children acquiring English as a second language from first to second grade. Journal of Educational Psychology, 101, 330-344. doi:10.1037/a0014320

Haenggi, D., \& Perfetti, C. (1992). Individual differences in reprocessing of text. Journal of Educational Psychology, 84, 182-192. doi:10.1037/0022-0663.84.2.182

Hasegawa, M., Carpenter, P. A., \& Just, M. A. (2002). An fMRI study of bilingual sentence comprehension and workload. NeuroImage, 15, 647-660. doi:10.1006/nimg.2001.1001

Keenan, J., \& Betjemann, R. (2006). Comprehending the gray oral reading test without reading it: Why comprehension tests should not include passage-independent items. Scientific Studies of Reading, 10, 363-380. doi:10.1207/s1532799xssr1004 2

Keenan, J., \& Brown, P. (1984). Children's reading rate and retention as a function of the number of propositions in a text. Child Development, 55, 1556-1569. doi:10.2307/1130026

Kintsch, W. (1974). The representation of meaning in memory. Hillsdale: Erlbaum. Retrieved from PsycINFO database.

Kintsch, W., \& Keenan, J. (1973). Reading rate and retention as a function of the number of propositions in the base structure of sentences. Cognitive Psychology, 5, 257-274. doi:10.1016/00100285(73)90036-4

Kintsch, W., Kozminsky, E., Streby, W. J., McKoon, G., \& Keenan, J. M. (1975). Comprehension and recall of text as a function of content variables. Journal of Verbal Learning and Verbal Behavior, 14, 196-214. doi:10.1016/S0022-5371(75)80065-X

Kintsch, W., \& van Dijk, T. (1978). Toward a model of text comprehension and production. Psychological Review, 85, 363 394. doi: $10.1037 / 0033-295 X .85 .5 .363$

Lee, J. F. (1986). On the use of the recall task to measure L2 reading comprehension. Studies in Second Language Acquisition, 8, 201212. doi: $10.1017 / \mathrm{S} 0272263100006082$

Lund, R. (1991). A comparison of second language listening and reading comprehension. Modern Language Journal, 75, 196204. doi: $10.2307 / 328827$

Meyer, B. J. F. (1974). The organization of prose and its effect on recall. Unpublished doctoral dissertation, Cornell University.
Miller, A. C., \& Keenan, J. M. (2009). How word reading skill impacts text memory: The centrality deficit and how domain knowledge can compensate. Annals of Dyslexia, 59, 99-113. doi:10.1007/s11881-009-0025-x

O’Brien, E. J., \& Myers, J. L. (1987). The role of causal connections in the retrieval of text. Memory \& Cognition, 15, 419-427.

Rawson, K., \& Kintsch, W. (2004). Exploring encoding and retrieval effects of background information on text memory. Discourse Processes, 38, 323-344. doi:10.1207/s15326950dp3803_3

Recht, D., \& Leslie, L. (1988). Effect of prior knowledge on good and poor readers' memory of text. Journal of Educational Psychology, 80, 16-20. doi:10.1037/0022-0663.80.1.16

Samuelstuen, M., \& Bråten, I. (2005). Decoding, knowledge, and strategies in comprehension of expository text. Scandinavian Journal of Psychology, 46, 107-117. doi:10.1111/j.14679450.2005.00441.x

Schmidt-Rinehart, B. (1994). The effects of topic familiarity on second language listening comprehension. Modern Language Journal, 78, 179-189. doi:10.2307/329008

Taglieber, L. K., Johnson, L. L., \& Yarborough, D. B. (1988). Effects of pre-reading activities on EFL reading by Brazilian college students. TESOL Quarterly, 22, 455-472.

Tudor, T. (1988). A comparative study of the effects of two prereading formats on L2 reading comprehension. RELC Journal, $19,71-87$.

Tyler, M. (2001). Resource consumption as a function of topic knowledge in nonnative and native comprehension. Language Learning, 51, 257-280. doi:10.1111/1467-9922.00155

van den Broek, P., \& Trabasso, T. (1986). Causal networks versus goal hierarchies in summarizing text. Discourse Processes, 9, 1-15. doi: $10.1080 / 01638538609544628$

Woodcock, R. W., McGrew, K. S., \& Mather, N. (2001). WoodcockJohnson III tests of achievement. Itasca: Riverside.

Wolff, D. (1987). Some assumptions about second language text comprehension. Studies in Second Language Acquisition, 9, $307-$ 326. doi: $10.1017 / \mathrm{S} 0272263100006707$

Wolf, D. (1993). A comparison of assessment tasks used to measure FL reading comprehension. Modern Language Journal, 77, 473489. doi: $10.2307 / 329673$ 